\title{
A Factor Linking Floral Organ Identity and Growth Revealed by Characterization of the Tomato Mutant unfinished flower development (ufd)
}

\section{OPEN ACCESS}

Edited by:

Catherine Anne Kidner,

University of Edinburgh, UK

Reviewed by:

Sinead Drea,

University of Leicester, UK Adrienne H. K. Roeder,

Cornell University, USA

*Correspondence: Rafael Lozano

rlozano@ual.es

${ }^{\dagger}$ Present Address: Muriel Quinet,

Groupe de Recherche en Physiologie végétale, Earth and Life Institute, Université Catholique de Louvain, Louvain-la-Neuve, Belgium

Specialty section:

This article was submitted to Plant Evolution and Development, a section of the journal Frontiers in Plant Science

Received: 05 August 2016 Accepted: 19 October 2016 Published: 07 November 2016

Citation:

Poyatos-Pertínez S, Quinet $M$ Ortiz-Atienza A, Yuste-Lisbona FJ, Pons C, Giménez E, Angosto T, Granell A, Capel $J$ and Lozano $R$ (2016) A Factor Linking Floral Organ Identity and Growth Revealed by Characterization of the Tomato Mutant unfinished flower development (ufd).

Front. Plant Sci. 7:1648 doi: 10.3389/fpls.2016.01648

\author{
Sandra Poyatos-Pertíñez ${ }^{1}$, Muriel Quinet ${ }^{1 \dagger}$, Ana Ortíz-Atienza ${ }^{1}$, \\ Fernando J. Yuste-Lisbona ${ }^{1}$, Clara Pons ${ }^{2}$, Estela Giménez ${ }^{1}$, Trinidad Angosto ${ }^{1}$, \\ Antonio Granell ${ }^{2}$, Juan Capel ${ }^{1}$ and Rafael Lozano ${ }^{1 *}$ \\ ${ }^{1}$ Centro de Investigación en Biotecnología Agroalimentaria, Universidad de Almería, Almería, Spain, ${ }^{2}$ Laboratorio de \\ Genómica de Plantas y Biotecnología, Instituto de Biología Molecular y Celular de Plantas, Consejo Superior de \\ Investigaciones Científicas, Universidad Politécnica de Valencia, Valencia, Spain
}

Floral organogenesis requires coordinated interactions between genes specifying floral organ identity and those regulating growth and size of developing floral organs. With the aim to isolate regulatory genes linking both developmental processes (i.e., floral organ identity and growth) in the tomato model species, a novel mutant altered in the formation of floral organs was further characterized. Under normal growth conditions, floral organ primordia of mutant plants were correctly initiated, however, they were unable to complete their development impeding the formation of mature and fertile flowers. Thus, the growth of floral buds was blocked at an early stage of development; therefore, we named this mutant as unfinished flower development (ufd). Genetic analysis performed in a segregating population of 543 plants showed that the abnormal phenotype was controlled by a single recessive mutation. Global gene expression analysis confirmed that several MADS-box genes regulating floral identity as well as other genes participating in cell division and different hormonal pathways were affected in their expression patterns in ufd mutant plants. Moreover, ufd mutant inflorescences showed higher hormone contents, particularly ethylene precursor 1-aminocyclopropane-1-carboxylic acid (ACC) and strigol compared to wild type. Such results indicate that UFD may have a key function as positive regulator of the development of floral primordia once they have been initiated in the four floral whorls. This function should be performed by affecting the expression of floral organ identity and growth genes, together with hormonal signaling pathways.

Keywords: flower development, organ growth, phytohormones, transcriptome, Solanum lycopersicum L., UFD gene

\section{INTRODUCTION}

Besides its importance as a major vegetable species, tomato (Solanum lycopersicum L.) has been considered as a model species for studying the genetic and hormonal regulation of reproductive development. However, most studies on this topic have focused on the development and ripening of tomato fruit (Lozano et al., 2009; Pesaresi et al., 2014; Azzi et al., 2015) and less attention has been 
given to the study of floral organogenesis compared to that given to other model plant species such as Arabidopsis, Antirrhinum, and rice. In Arabidopsis, once the floral transition takes place, the shoot apical meristem (SAM) acquires an inflorescence meristem (IM) fate allowing the formation of floral meristems (FM) on its flanks. Genetic control of FM initiation includes, as a main measure, the acquisition of the floral identity by the activation of the FM identity genes LEAFY (LFY) and APETALA1 (reviewed in Siriwardana and Lamb, 2012; Winter et al., 2015). Tomato plants exhibit a sympodial growth pattern where the SAM converts into an IM, and vegetative development continues from a specialized axillary meristem called sympodial meristem, which arises from the axil of the last leaf. Meanwhile, the IM produces a new IM on its side before differentiating into a FM. Reiteration of IMs that are arranged perpendicular to one another, results in the production of an inflorescence organized in a zig-zag pattern (reviewed in Lozano et al., 2009; Thouet et al., 2012). FALSIFLORA has been characterized as the LFY ortholog in tomato where it plays similar functions as $L F Y$ as a regulator of FM identity (Molinero-Rosales et al., 1999; Thouet et al., 2012). However, MACROCALYX (MC), the tomato AP1 homolog, most likely regulates IM instead of FM fate in tomato (Vrebalov et al., 2002; Yuste-Lisbona et al., 2016).

Once FM has been determined, floral organ identity is established from specific meristematic domains, which result in the initiation of sepal, petal, stamen, and carpel primordia in the four whorls forming the flowers of most angiosperms. This process is regulated by several overlapping gene functions which act in accordance with the $\mathrm{ABC}(\mathrm{DE})$ model (Coen and Meyerowitz, 1991; Causier et al., 2010). Most of the ABC identity functions are carried out by members of the homeotic MADSbox gene family. They encode transcriptional factors, which form part of multimeric protein complexes capable of binding to the target genes responsible for the morphological features of mature floral organs (Honma and Goto, 2001; Smaczniak et al., 2012). In tomato, phenotypic characterization and expression pattern analyses performed in floral homeotic mutants and transgenic plants with affected MADS-box gene expression seem to confirm the $\mathrm{ABC}(\mathrm{DE})$ model. Thus, the $M C$ A-class gene is, amongst others, involved in sepal development (Vrebalov et al., 2002; Yuste-Lisbona et al., 2016). B-class gene function is carried out by two paralogous of APETALA3, i.e., Tomato AP3 [TAP3; syn. STAMENLESS (SL)] and Tomato MADS box 6 (TM6), and two PISTILLATA (PI) homologs, i.e., Tomato PISTILLATA (TPI; syn. SlGLO2) and S. lycopersicum GLOBOSA (SlGLO; syn. SlGLO1, LePI, TPIB; Pnueli et al., 1991; Kramer et al., 1998; Busi et al., 2003; de Martino et al., 2006; Leseberg et al., 2008; Geuten and Irish, 2010; Quinet et al., 2014). The TOMATO AGAMOUS 1 (TAG1) gene specifies stamen and carpel identity (Pnueli et al., 1994a) and together with TOMATO AGAMOUS LIKE-1 (TAGL1; syn. ARLEQUIN (ALQ) (Vrebalov et al., 2009; Giménez et al., 2010), represent C-class gene function, while TM5 (Pnueli et al., 1994b) and TM29 (syn. TAGL2; Ampomah-Dwamena et al., 2002; Busi et al., 2003) have been proposed as E-class genes. The TAGL11 gene represents D class gene function in tomato and is involved in flower and mainly in fruit development (Busi et al., 2003).
Floral organ development includes not only the meristematic signals of the $\mathrm{ABC}(\mathrm{DE})$ network which indicate the correct place where a tissue or organ will differentiate from the FM, but also changes in cell proliferation leading to the growth of floral organ primordia. This program initiates in response to meristematic signals and interacts with the organ identity genes (Reinhardt, 2005; Dornelas et al., 2011; Wellmer et al., 2014). As a result, each organ primordia grows initially by cell division and, subsequently, by cell expansion until it acquires its final size and shape (Anastasiou and Lenhard, 2007; Bögre et al., 2008). Members from the ovate family protein (OFP) have been characterized as regulators of cell proliferation. Thus, OVATElike proteins act as transcriptional repressors in Arabidopsis, mainly affecting the expression of GIBBERELIN 20 OXIDASE 1, a key player in the gibberellin biosynthesis, causing a reduction in cell elongation (Hackbusch et al., 2005; Wang et al., 2007, 2011). In tomato, the OVATE gene acts as a negative regulator of growth, since the loss-of-function ovate mutant exhibits elongated carpels (Liu et al., 2002). Likewise, significant changes in cell division are due to a mutation in the tomato cell cycle-control gene FRUIT WEIGHT 2.2 (FW2.2), which encodes a negative regulator of this process (Frary et al., 2000). On the contrary, SUN is a member of the IQD family of calmodulin-binding proteins and acts as a positive regulator of growth in tomato, since its high expression level is associated with increases in cell division (Xiao et al., 2008, 2009).

Phytohormones participate in the initiation and outgrowth of floral organ primordia such as auxins that play a key role in the arrangement of floral organs (Wellmer et al., 2014). The unraveling of the genetic network and molecular mechanisms which control the dynamics of flower development, and particularly, the link between identity and growth of floral organs, is an important goal of plant biology currently (Jaeger et al., 2013) and some of the players involved in this process have begun to be identified in Arabidopsis (reviewed in Dornelas et al., 2011; ÓMaoiléidigh et al., 2014; Wellmer et al., 2014). Extensive genetic and molecular analyses have shown that the homeotic MADS box proteins involved in the ABC model play a master role during both organ identity determination and organ morphogenesis (reviewed in Dornelas et al., 2011; Wellmer et al., 2014). Recently, genome-wide approaches have led to substantial progresses in the identification of genes functioning downstream in the signaling pathway triggered by MADS box proteins (Wellmer et al., 2004, 2006; Kaufmann et al., 2009, 2010; Yant et al., 2010; Wuest et al., 2012; Ó’Maoiléidigh et al., 2013; Pajoro et al., 2014). Many of these genes code for transcription factors or are involved in hormone biosynthesis and signaling (Dornelas et al., 2011; Wellmer et al., 2014). Thus, the floral organ identity factors appear to mediate floral organogenesis by controlling the expression of other regulatory genes, whereas it is though that different phytohormones have a key function in the initiation and differentiation of floral organs. Most phytohormones were indeed shown to be involved in floral organ development in Arabidopsis (Chandler, 2011; Yuan and Zhang, 2015; Marsch-Martínez and de Folter, 2016). Auxin, gibberellin, and jasmonic acid are involved in petal development through the regulation of the BIGPETAL transcription factor (Chandler, 
2011). During stamen development, jasmonic acid interacts with auxins and gibberellins to regulate anther development and pollen maturation (Plackett et al., 2011; Yuan and Zhang, 2015). Gibberellins also control filament elongation (Plackett et al., 2011). In addition to these major regulators of stamen development, ethylene has been shown to be involved in stamen initiation and cytokinins and brassinosteroids are required for proper pollen development (Chandler, 2011). Regarding gynoecium development, auxins promote carpel initiation, gynoecium growth, and proper style and stigma formation while cytokinins participate in the development of the carpel margin meristem and derived tissues as well as in the valve margin formation (Marsch-Martínez and de Folter, 2016). Finally, after fertilization, synthesis, and signaling of gibberellins are induced to trigger fruit growth (Gallego-Giraldo et al., 2014). However, although progress has been made in the recent years in the understanding of the hormonal control of floral organ development, we are not yet at the stage of having precise networks between hormone signaling pathways and floral organ identity and building gene interactions for the different floral organ developmental stages.

The genetic mechanisms underlying floral organ growth remain largely unknown, particularly in tomato, where genes specifically involved in this developmental process have not been discovered hitherto. The tomato unfinished flower development ( ufd) mutant has been identified from an EMS-mutagenized M2 population of tomato (cv. Moneymaker). The ufd flowers are unable to complete the normal growth of floral organ primordia, even though they were correctly initiated in their corresponding floral whorls. We recently characterized the genetic interactions of $u f d$ with other tomato mutants showing defects in diverse processes related to inflorescence and flower development, which showed that UFD might play a pivotal role between inflorescence architecture and flower initiation genes (Poyatos-Pertíñez et al., 2016). In this paper, we further characterized $u f d$ mutant to gain insight in the role of UFD during reproductive development of tomato. Transcriptome analysis indicated that UFD has a key role in the genetic control of floral organogenesis by affecting the expression of floral organ identity and cell cycle genes, and plant hormones. To provide additional insights into the regulatory interactions of UFD with floral organ identity and growth genes, expression analyses were carried out in $u f d$ and wild type (WT) flowers during inflorescence development. Moreover, the complete hormonal profile was investigated in $u f d$ flowering shoot apices to highlight the involvement of UFD in hormonal metabolism and the role of hormones in tomato flower development. All together our results indicated that UFD has a key function as positive regulator of floral organ identity and growth, presumably by regulating transcriptional activity and hormonal signaling pathways.

\section{MATERIALS AND METHODS}

\section{Plant Material and Growth Conditions}

The $u f d$ mutant was isolated from an M2 population of tomato (S. lycopersicum L., cv. Moneymaker), which was generated by mutagenesis with ethyl methanesulphonate (EMS). Genetic analysis of $u f d$ mutation was initially performed on 20 plants of the original M2 family, and subsequently in a population composed of 543 M3 plants obtained by selfing a single heterozygous M2 plant (genotype+/ufd). Phenotype segregations were verified by a $\chi^{2}$-test.

Seeds were germinated in plastic pots filled with sphagnum, peat/coco, peat/vermiculite substrate mixture (3:2:1). Plants were grown under standard greenhouse conditions, $25^{\circ} \mathrm{C}$ daytime maximum to $10^{\circ} \mathrm{C}$ night minimum temperature under $\sim 14 \mathrm{~h}$ natural light. All plants received regular watering and fertilizer treatments.

\section{Microscope Analysis}

Fresh plant tissues (inflorescences, flowers, floral buds, and floral organs) were dissected and examined with a Nikon SMZ445 stereomicroscope to determine morphological and developmental characteristics. For histological studies, inflorescence samples were harvested and treated in FAA $(3.7 \%$ formaldehyde, $5 \%$ acetic acid, $50 \%$ ethanol) for $2 \mathrm{~h}$ under partial vacuum conditions and then kept for $20-24 \mathrm{~h}$ at room conditions. For light microscopy, fixed samples were dehydrated in an ethanol series (70-100\%) and embedded in paraffin (Paraplast plus, Sigma-Aldrich). Sections ( $8 \mu \mathrm{m}$-thick) were cut with a Leica RM2145 microtome and stained using eosin. Later, paraffin was removed and tissue sections were dehydrated in an ethanol series, stained in a $1 \%$ Blue Toluidine solution and analyzed and photographed with a Nikon Optiphot2 microscope. Scanning electron microscopy (SEM) analysis was carried out as follows: samples were fixed in FAEG (3.7\% formaldehyde, $5 \%$ acetic acid, 50\% ethanol, and $0.5 \%$ glutaraldehyde) and kept in $70 \%$ ethanol. Samples were dehydrated in an ethanol with increasing concentrations (70-100\%) and them critical-point dried in liquid $\mathrm{CO}_{2}$. Subsequently, samples were gold coated and observed using a Hitachi S-3500N scanning electronic microscopy machine at an accelerating voltage of $10 \mathrm{kv}$.

\section{Transcriptome Analysis by Microarray Hybridization}

In order to examine the differential gene expression patterns in wild type and ufd inflorescences, RNA samples were obtained from pooled flowering shoot apices of the last initiated inflorescence harvested on 40-day-old plants of each wild type and $u f d$ genotypes, in which the oldest flower buds were around stage 4 (according to Brukhin et al., 2003). Three independent biological replicates per genotype were performed, each one with at least 20 inflorescence apices (4-5 mm long). The six RNA samples were reverse transcribed and labeled with Cy5. Equal aliquots of RNA from the six different samples were mixed and labeled with $\mathrm{Cy} 3$ to create a common reference RNA sample. An aliquot of the common reference sample was mixed with each labeled test cDNA sample and the subsequent probes were then hybridized to TOM2 oligo-arrays as previously described (Lytovchenko et al., 2011). The same reference sample was used to hybridize each slide, which enabled the direct comparison of all hybridization experiments. Raw data files are available with accession number E-MTAB-5144 at ArrayExpress (www. ebi.ac.uk/arrayexpress/). The mean LOWESS-normalized values 
for a gene between wild type and $u f d$ samples was compared by one-way ANOVA in order to identify differentially expressed genes. An adjusted $P$-value cut-off of 0.05 was used to identify genes as differentially expressed between experimental groups. A hierarchical clustering was constructed using Pearson correlation coefficient as a similarity metric and the logarithm ratios of the fold change values for each gene in each experimental group as the input data.

\section{Quantitative Real-Time PCR Analysis}

Gene expression analyses were performed by quantitative realtime PCR (qRT-PCR) experiments on wild type and mutant inflorescences at three stages of development: (i) flowering shoot apex containing the last initiated inflorescence, where the oldest flower buds was around stage 4 (according to Brukhin et al., 2003); (ii) type 1 inflorescence (IF1) corresponded to the penultimate initiated inflorescence, including flower buds from stages 6 to 12 in WT and up to stage 5 in $u f d$, since the growth of floral buds is arrested at this stage; and (iii) type 2 inflorescence (IF2) that is the third inflorescence from the top, which contained floral buds from stages 8-18 in WT while in the ufd IF2 only included flower buds up to stage 5. Total RNA was isolated using the Trizol Kit (Invitrogen) according to the manufacturer's recommendations. Contaminating DNA was removed using the DNA-free ${ }^{\mathrm{TM}}$ kit (Ambion). Subsequently, M-MLV Reverse transcriptase kit (Invitrogen) was used to synthesize first strand cDNA. The reaction was performed according to the recommended protocol by using an oligo (dT) primer and $1 \mu \mathrm{g}$ RNA. Specific primers for each examined gene are described in Supplementary Table 1. SYBR Green PCR Master Mix kit (Applied Biosystems) was used to perform qRT-PCR reactions in a 7300 Real-Time PCR System (Applied Biosystems), according to the manufacturer's instructions. For each inflorescence stage, qRT-PCRs with three biological and two technical replicates were performed. Seven thousand and three hundred System Sequence Detection Software v1.2 (Applied Biosystem) was used for data collection and analysis of expression levels. Results were processed using the $\Delta \Delta \mathrm{Ct}$ calculation method, expressed in arbitrary units and normalized by comparison to the housekeeping UBIQUITINE3 (UBI3) gene and then to the value of the WT control. A Student's $t$-test was used for the comparison of data sample means and probability values $<0.05$ were considered statistically significant $(P<0.05)$.

\section{Hormonal Analysis}

Concentrations of the endogenous phytohormones including cytokinins, auxins, gibberellins, abscisic acid, salicylic acid, jasmonic acid, ethylene precursor 1-aminocyclopropane-1carboxylic acid (ACC), brassinosteroids, benzoic acid, and their metabolites were determined in developing inflorescences (pooled flowering shoot apices harvested on 40-day-old plants) of $u f d$ and WT plants according to Quinet et al. (2014). Phytohormones were extracted using 50-100 mg lyophilised plant material from three biological replicates with methanol/formic acid/water (15:1:4, by volume) and then purified using the dual-mode solid-phase method (Dobrev and Kamínek, 2002). An HPLC Ultimate 3000 (Dionex,
Sunnyvale, CA, USA) coupled to a 3200 Q TRAP hybrid triple quadrupole/linear ion trap mass spectrometer (Applied Biosystems, Foster City, CA, USA) was used to perform the analysis and quantification of plant hormone levels by means of a multilevel calibration graph with $2 \mathrm{H}$-labeled internal standards. Shapiro-Wilk and Levene's-test were applied to evaluate the normality and homogeneity of the results, respectively (data were transformed when required). ANOVA and Student's-test were used for statistical analysis to evaluate the significance of the genotype effects on the hormonal composition using SAS 9.2.

\section{RESULTS}

\section{Phenotype and Genetic Characterization of ufd Mutant}

A screening was performed in an ethyl methanesulfonate (EMS)induced tomato mutant collection with the aim to isolate new regulatory genes involved in flower development, particularly focused on those mutant phenotypes affected in floral organ identity and growth. As result, a mutant was identified and named $u f d$ as both their inflorescences and flowers showed a severe reduction in size and were unable to complete their normal development (Figure 1). As wild type tomato plants (cv. Moneymaker), the primary shoot of ufd mutant plants developed an initial vegetative segment composed by 6-7 leaves before the formation of the first inflorescence, and the subsequent plant growth continued through the development of an indeterminate number of sympodial segments, which were normally composed of three leaves and one inflorescence (Figures 1A-C,E). No differences between $u f d$ and WT plants were observed in vegetative growth and flowering time (Figure 1 and Supplementary Table 2). After undergoing floral transition, ufd plants initiated the development of the inflorescence by the formation of the successive flowers in a zig-zag pattern, as normally occur in wild type tomato plants (Figures 1D,F). Nevertheless, once floral buds were initiated, they were unable to follow a normal growth thus impeding the formation of mature flowers. In addition, the $u$ fd plants yielded a smaller number of flowers per inflorescence compared to the WT plants (5.21 \pm 0.89 and $7.83 \pm 0.89$ flowers in $u f d$ and WT, respectively, $P<0.05$ ).

A genetic analysis was performed in an M2 segregating population, where the $u f d$ mutant phenotype was observed in one quarter of plants (15 WT: $5 \mathrm{ufd}$ ), suggesting a monogenic and recessive inheritance pattern. As the mutation resulted in sterile plants, WT individuals of the same M2 family were selfed, and an M3 segregating population was obtained from a single heterozygous M2 individual. Phenotypic characterization of 543 plants of this M3 progeny revealed a 3:1 ratio for the wild type: mutant phenotype (391: $152, \chi^{2}=2.59, P=0.11$ ), which corroborated that $u f d$ phenotype was caused by a monogenic and recessive mutation.

\section{Floral Ontogeny of ufd Mutant}

A detailed analysis of floral organogenesis (Figure 2) was performed on floral buds of $u f d$ and WT plants at different developmental stage by SEM, according to those described by Brukhin et al. (2003). At early stages (from stage 3 to 5), ufd floral 

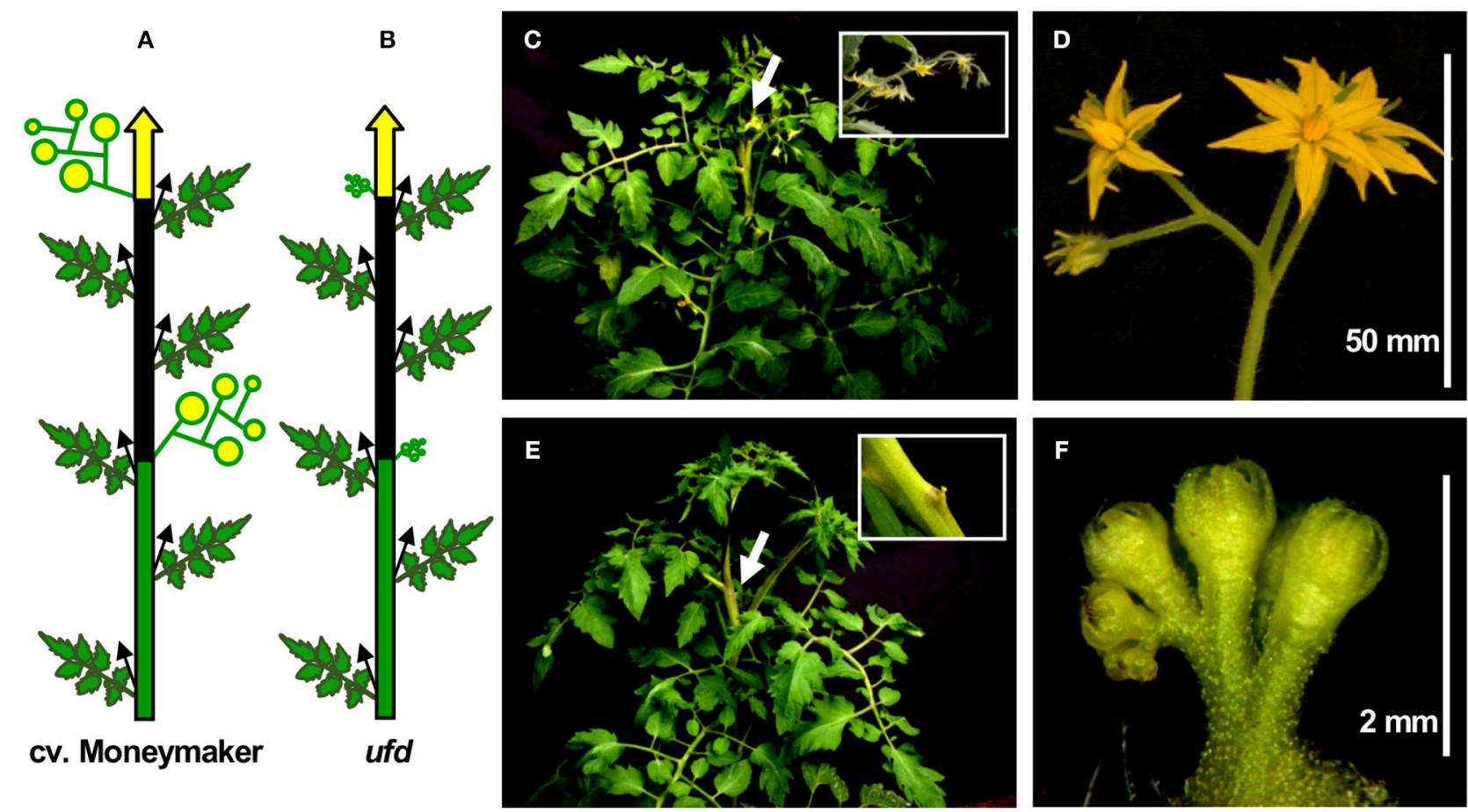

FIGURE 1 | Structure of wild type and unfinished flower development (ufd) plants grown under standard conditions. (A,B) Diagrammatic representation of wild type (WT) and mutant tomato plant growth. Green bars represent initial vegetative segment. Sympodial segments are in black. Yellow arrows represent sympodial meristems. Circles symbolize flowers, and black arrows represent developed axillary shoots. (C,D) Morphology of a wild type (C) and an ufd mutant plant (E). White arrows indicate inflorescence position. The insets show a closer view of the inflorescences. (D,F) Adult inflorescences of the wild type (D) and the ufd mutant (F). White bars show inflorescence size.

buds were found to develop similarly to those of the wild type background (Figures 2A,B,F,G). As the development progressed, organ primordia were initiated at the expected positions in the corresponding floral whorls. Initially, sepal primordia arose in a helical order. Subsequently, five to six petal primordia appeared simultaneously, followed by stamen and carpel primordia, which alternated with respect to the preceding whorl primordia as described previously (see review of Lozano et al., 2009). Slightly later, and coinciding with the end of stage 5 and stage 6 of tomato flower development (Figures 2C,H), the growth of floral buds is blocked in ufd plants (Figures 2I,J) while WT flowers continued to develop (Figures 2D,E), therefore this was considered the "most adult stage" of $u f d$ flower development. At this stage, sepal primordia forming the first whorl ceased their development when they were still fused (Figures $\mathbf{2 H}, \mathbf{I}$ ), and trichomes appeared in a lower density on the sepal abaxial surface but restricted to a reduced area around the apical zone (Figures 2D,I). Petal primordia (second whorl) also stopped their development and remained as fused organs as expected for this developmental stage. They did not elongate enough to cover stamen primordia and trichomes were not observed on their abaxial surface (Figure $\mathbf{2 H}$ ). The third floral whorl in adult ufd flowers consisted of dome-shaped stamen primordia, which reached a size comparatively higher than the petals, while carpel primordia led the formation of one or two faint cavities in the innermost whorl (Figure 2H). Moreover, no morphological differences were found in the epidermal cells of mutant floral organs or in their whorled disposition, indicating that ufd mutation does not affect morphology or phyllotaxis of floral organs (Figures 2D,E, I,J).

\section{Transcriptome Analysis of ufd Mutant}

To have a wider perspective of the transcriptional changes promoted by the ufd mutation during floral development, a global gene expression analysis was performed using the tomato-TOM2 microarray. With this purpose, each of the three biological replicates of the wild type and $u f d$ transcriptomes was compared against a common reference sample. After filtering out redundant spots and selecting the ones which were significantly expressed between any of the two samples, a total of 325 transcripts were found to show differential expression in their mRNA levels in $u f d$, corresponding to $4.4 \%$ of all spots (7380) which passed quality controls.

The differentially expressed genes (DEGs) identified from the $u f d$ vs. WT comparison were assigned to different functional categories (Supplementary Figure 1, Supplementary Tables 3, 4) according to the Tomato Functional Genomics Database (TFGD, http://ted.bti.cornell.edu/). Cellular component categories such as cell, intracellular, and organelle were more represented in the up-regulated gene dataset while the photosystem and 

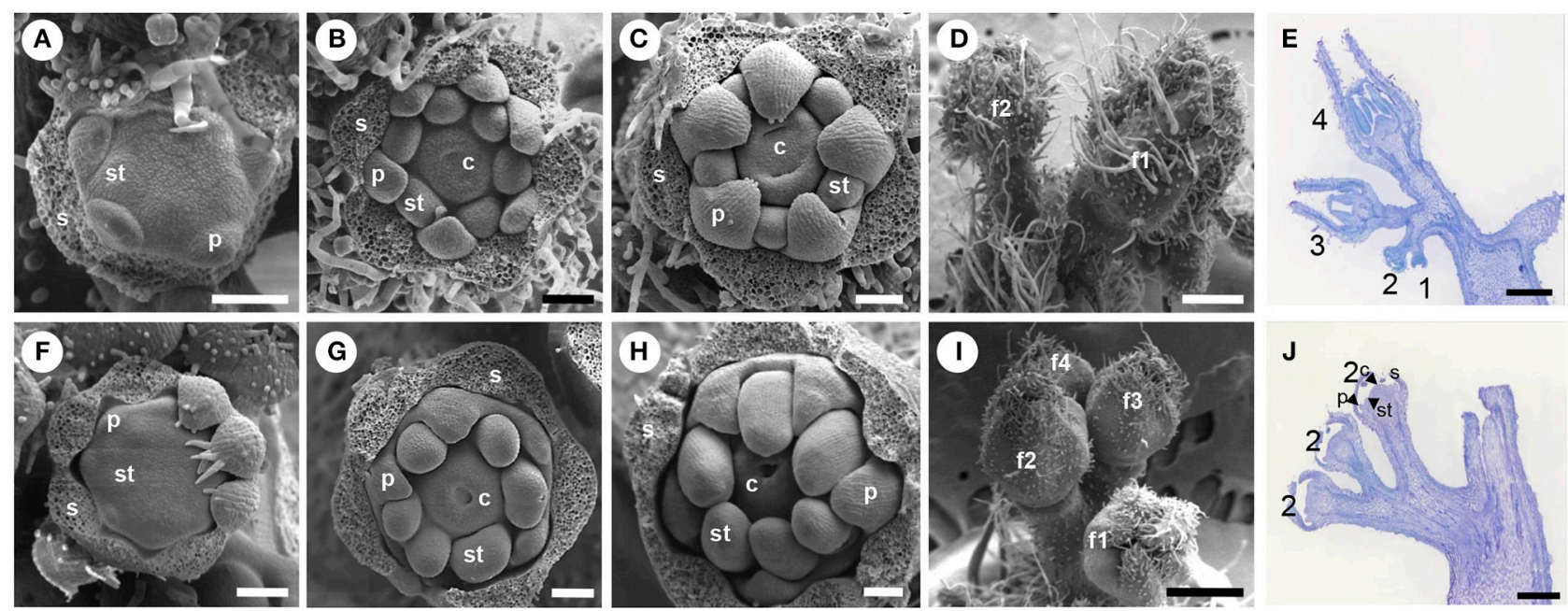

FIGURE 2 | Scanning electron microscopy (SEM) analysis and histological microscopy of floral development from wild type and unfinished flower development (ufd) mutant plants. (A-E) Wild type floral buds. (F-J) Mutant flowers. Wild type (A) and ufd (F) floral buds at stage 3, corresponding to stamen primordia initiation (most sepals were removed). Wild type (B) and ufd (G) floral buds at stage 5, when carpel primordia emerge and the distinct ovary cavities are visible (sepals removed). (C) Wild type flower at stage 6, when petals bend down to cover reproductive organs and the carpels grow up but are still unfused (sepals removed). (H) Floral bud at the most adult stage observed in ufd mutant plants (sepals removed). (D) Wild type inflorescence with floral buds at stage 6 (f1) and 5 (f2). (I) ufd inflorescence showing the most developed floral bud at stage 5 (f1) and others at earlier stages (f2-f4). (E) Wild type inflorescence with flowers at different development stages (1, 2, 3, and 4). (J) ufd inflorescence with all flowers showing the same development stage (floral organs stop their development at primordia stage). All developmental stages are classified according to Brukhin et al. (2003). s, sepal; p, petal; st, stamen; c, carpel. Scale bars are $200 \mu \mathrm{m}$ (A-C,F-H), $500 \mu \mathrm{m}$ (D,E,I,J).

thylakoid-light-harvesting complex categories were enriched in the down-regulated gene dataset (Supplementary Figure 1A, Supplementary Tables 3,4). With regard to their molecular function, the percentage of transcripts classified under categories linked to nucleic acid binding, structural constituent of ribosome, and structural molecule activity were greater in the up-regulated gene dataset (Supplementary Figure 1B, Supplementary Table 3). Categories such as transcription regulator activity, transcription factor activity, and sequencespecific DNA binding were more richly represented in the downregulated gene dataset (Supplementary Figure 1B, Supplementary Table 4).

In order to identify genes sharing a similar expression pattern, the whole set of 325 DEGs was clustered into eight clusters (numbered 1-8) according to their differential expression using the Pearson clustering algorithm (Figure 3 and Supplementary Table 5). DEGs in ufd samples were grouped into two large clusters (clusters 1 and 2) and several smaller clusters. The largest cluster (cluster 1,29\%) included genes repressed both in wild type and $u f d$ mutant samples when compared with the common reference, although at a lower level in the later one. Among others, this cluster included genes encoding transcription factors belonging to WRKY and bHLH families, and others involved in the ethylene pathway such as ETHYLENE RESPONSE FACTOR 1 and Lycopersicum esculentum Jasmonic Acid 2 (LEJA2). Cluster 2 $(20 \%)$ and cluster $4(6 \%)$ included genes showing minor downregulation in the WT and slight up-regulation in the mutant, although the former showed either greater down-regulation in the WT while the latter showed greater up-regulation in the mutant. Cluster 2 included the elongation factor 1-alpha and some transcription factors, whereas cluster 4 included genes encoding transcription factors of AP2 and WRKY families, together with the OVATE gene involved in tomato fruit shape and several ethylene-related genes. Cluster $3(10 \%)$ consisted of mostly constitutive genes showing a similar expression profile to that of cluster 2 and cluster 4 . Some representatives of this cluster were genes encoding $\mathrm{H} 2 \mathrm{~A}$ and $\mathrm{H} 2 \mathrm{~B}$ histones and protein kinases. Genes included in cluster 7 (14\%) showed major up-regulation in wild type inflorescences and minor up-regulation in the mutant ones. Some floral organogenesisspecific genes most of them belonging to the MADS-box family, such as TDR4, TDR6, LeMADS1, TM29, and putative orthologs of CRABS CLAW (CRC) and PMADS4 were found in this cluster, together with putative orthologs of AUXIN RESPONSE FACTOR 8 (ARF8), ARF6, and the PHYTOENE SYNTHASE gene involved in the synthesis of carotenoids. Cluster 5 (5\%) were also represented by genes up-regulated both in wild type and $u f d$ inflorescences, although differences in the expression level favored the ufd inflorescences in this cluster, which was mostly composed by constitutive genes encoding ribosomal proteins.

The loss-of-function phenotype of the ufd mutant, which agreed with its recessive inheritance, led us to focus our attention on two additional clusters (Figure 3 and Supplementary Table 5). Cluster 8 (11\%) included genes significantly up-regulated in wild type inflorescences but strongly down-regulated in ufd mutant ones, among them those encoding MADS-box transcription factors such as $M C$ and a SlGLO1, as well as other putative 


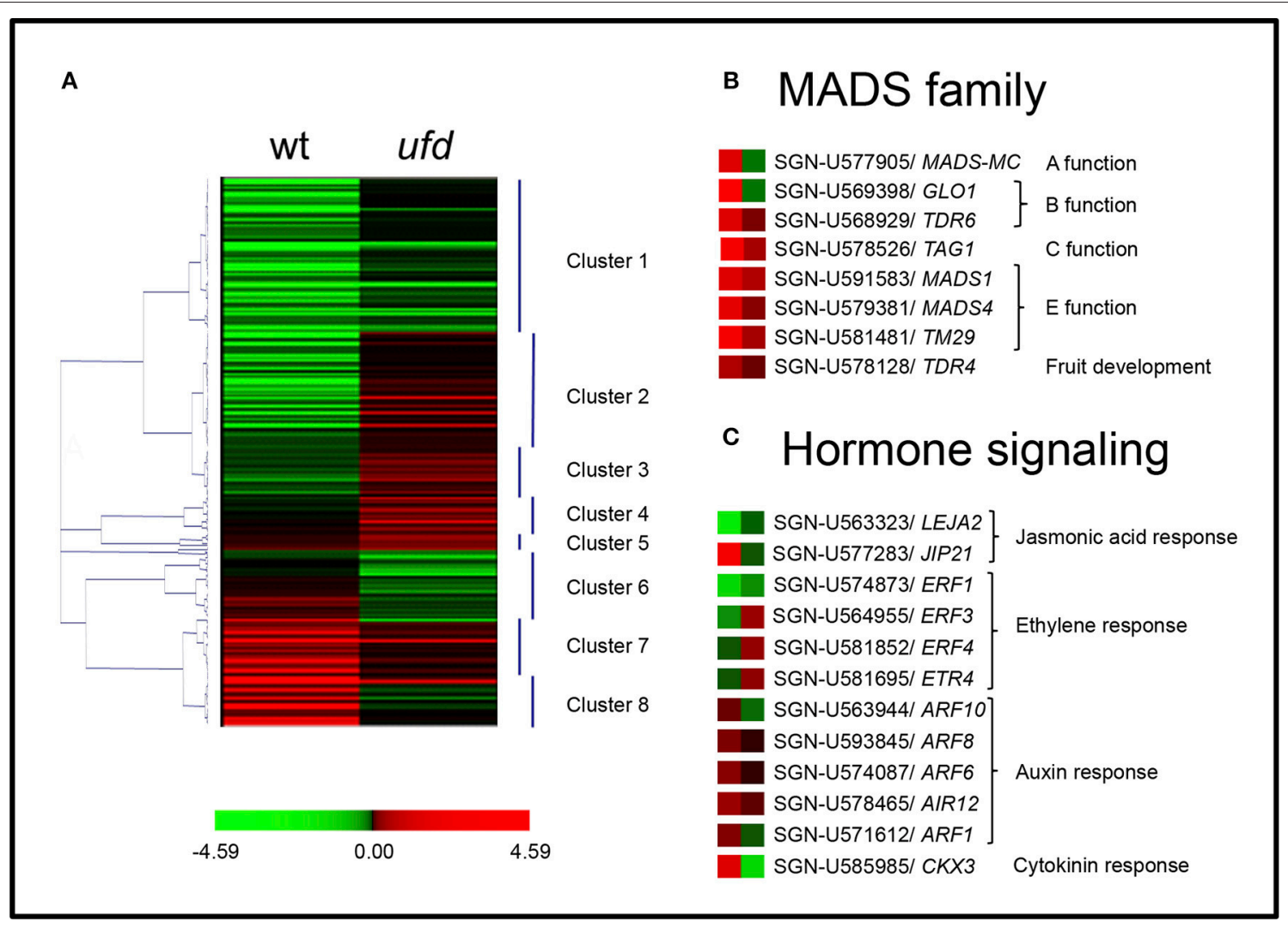

FIGURE 3 | Expression distribution of genes differentially expressed in unfinished flower development (ufd) and wild type (WT) tomato flowering shoot apices. (A) Hierarchical clustering of all genes differentially expressed in the ufd mutant compared to WT (see also Supplementary Table 5). (B,C) Heat-map of MADS genes and genes involved in hormone signaling, respectively. Color scale indicates logarithm ratio of the fold change in mutant compared with WT. Red color represents genes showing higher expression level and green color indicates reduced expression.

orthologs of flowering-related genes as CYTOKININ OXIDASE 3 (CKX3), ARGONAUTE, ARF1 and an ethylene-responsive transcription factor RAP2. Similarly, cluster $6(5 \%)$ contained genes, which were also up-regulated in WT, although at a lower level than in cluster 8, and greatly down-regulated in the ufd mutant. Some genes involved in abiotic stresses and in auxin response such as $A R F 10$ were included in this cluster.

Among the 325 DEGs, a total of 31 and 13 showed more than two-fold up- and two-fold down-regulation in the ufd mutant transcriptome, respectively (Table 1). The retrieved set of 31 up-regulated genes appeared not to be significantly enriched in any particular biological or molecular functional category, neither that corresponding to transcriptional regulatory nor developmental processes (Table 1). In this set four transcription factors were encountered, three of them belonging to the WRKY family together with the putative ortholog of the BRASSINOSTEROID ENHANCED EXPRESSION 3 bHLH transcription factor. Genes also included in this set code for an elongation factor-1 alpha, a calcium-dependent protein kinase, a cytochrome $\mathrm{P} 450$, a 2-oxoglutarate-dependent dioxygenase involved in ethylene synthesis, and LEJA2. Most of the remaining up-regulated genes were involved in either biotic or abiotic stress responses. The set that was downregulated in $u f d$ inflorescences was particularly enriched in genes encoding for transcription factors of the MADS-box family (Table 1), including SlGLO1, TM29, MC, TDR6, and a putative ortholog of $p M A D S 4$. An additional transcription factor encountered was the putative ortholog of Nicotiana tabacum CRC. The rest of the down-regulated genes included CKX3, chymotrypsin inhibitor jasmonic-induced protein 21 (JIP1), flower-specific gamma-thionin-like protein/acidic protein precursor and wound-induced proteinase inhibitor 1 .

All together, these results reveal that UFD may particularly regulate genes coding for transcription factors required for floral organ development and genes involved in hormone signaling (Figure 3). Some of these genes were investigated by qRT-PCR in order to confirm microarray expression data and to better characterize the UFD function.

\section{Expression of Genes involved in Floral Organ Identity and Growth}

The expression of floral organ identity genes $M C$ (A-class), SL (B-class), TAG1, and TAGL1 (C-class) and TM5, and TM29 (E-class) were analyzed by qRT-PCR in ufd mutant inflorescences containing floral buds at increasing developmental 
TABLE 1 | Differentially expressed genes showing more than two-fold up- and two-fold down-regulation in unfinished flower development (ufd) relative to wild type plants.

\begin{tabular}{|c|c|c|c|c|c|}
\hline Probe ID & Gene ID & Fold change & GenBank ID & Annotation & e-value \\
\hline LE9L13 & SGN-U579680 & 30.718 & P10798 & Ribulose bisphosphate carboxylase small chain 3B & $1 e-32$ \\
\hline LE23F03 & SGN-U582797 & 8.938 & BAA13150 & NT16 polypeptide (Nicotiana tabacum) & $2 \mathrm{e}-32$ \\
\hline LE15E11 & SGN-U571964 & 8.381 & AAF63515 & TMV-induced protein I (Capsicum annuum) & $2 e-71$ \\
\hline LE26P24 & SGN-U580191 & 8.125 & P17786 & Elongation factor 1-alpha & 0 \\
\hline LE14013 & SGN-U593442 & 4.746 & AAP43673 & PR5-like protein (Lycopersicon esculentum) & $1 e-77$ \\
\hline LE9D23 & SGN-U589805 & 4.206 & CAO39940 & Unknown protein & $2 e-19$ \\
\hline LE4M08 & SGN-U578841 & 3.925 & Q43502 & Proteinase inhibitor type II CEVI57 & $2 e-106$ \\
\hline LE6G19 & SGN-U580000 & 3.867 & AAG16757 & Putative glutathione S-transferase T2 (Lycopersicon esculentum) & $1 e-117$ \\
\hline LE13C07 & SGN-U576746 & 3.402 & AEC07585 & $\mathrm{C} 2 \mathrm{H} 2$ zinc finger protein FZF (Arabidopsis thaliana) & $8 e-94$ \\
\hline LE7I09 & SGN-U578506 & 3.396 & No hits & No hits & \\
\hline LE3I18 & SGN-U581493 & 3.361 & No hits & No hits & \\
\hline LE29C09 & SGN-U563323 & 3.341 & AF011555 & LEJA2 Jasmonic acid 2 & 0 \\
\hline LE31P05 & SGN-U570189 & 3.304 & AEC08624 & GCN5-related N-acetyltransferase-like protein (Arabidopsis thaliana) & $3 e-47$ \\
\hline LE28P15 & SGN-U567304 & 3.295 & AAK07676 & Non-symbiotic hemoglobin class 1 (Lycopersicon esculentum) & $2 \mathrm{e}-080$ \\
\hline LE6O08 & SGN-U577557 & 3.269 & AAU95238 & Osmotin-like protein (Solanum phureja) & $4 e-156$ \\
\hline LE25D18 & SGN-U577356 & 3.057 & No hits & No hits & \\
\hline LE26A07 & SGN-U579236 & 2.922 & BAD98961 & 2-oxoglutarate-dependent dioxygenase (Solanum lycopersicum) & 0 \\
\hline LE17C09 & SGN-U579850 & 2.728 & AEE73926 & Endoribonuclease Dicer-like 2 (Arabidopsis thaliana) & $5 e-120$ \\
\hline LE8C19 & SGN-U580303 & 2.710 & NP_849875 & MLP-like protein 28 (Arabidopsis thaliana) & $1 e-68$ \\
\hline LE17B14 & SGN-U571844 & 2.702 & AAA65637 & Peroxidase & 0 \\
\hline LE26L14 & SGN-U581493 & 2.697 & No hits & No hits & \\
\hline LE31K04 & SGN-U580119 & 2.573 & No hits & No hits & \\
\hline LE31O23 & SGN-U583039 & 2.514 & NP_177524 & BEE3, bHLH transcription factor (Arabidopsis thaliana) & $1 e-46$ \\
\hline LE22P06 & SGN-U571844 & 2.496 & AAA65637 & Peroxidase (Solanum lycopersicum) & 0 \\
\hline LE23B10 & SGN-U580500 & 2.428 & BAC23031 & WRKY-type DNA binding protein (Solanum tuberosum) & $3 e-82$ \\
\hline LE33C13 & SGN-U576561 & 2.351 & NP_189542 & Calcium-binding EF-hand family protein (Arabidopsis thaliana) & $9 e-19$ \\
\hline LE6l12 & SGN-U571983 & 2.229 & O64882 & Beta-glucosidase 17 & $2 e-171$ \\
\hline LE17F23 & SGN-U577821 & 2.225 & Q05047 & Cytochrome P450 (Catharanthus roseus) & $4 e-130$ \\
\hline LE18L09 & SGN-U580535 & 2.033 & ABM06179 & Glutathione transferase, putative & $3 e-41$ \\
\hline LE18E04 & SGN-U583014 & 2.020 & BAA89235 & WRKY-type DNA binding protein, TMV response-related (Nicotiana tabacum) & $4 e-89$ \\
\hline LE3G12 & SGN-U596360 & 2.003 & NP_174279 & WRKY71; transcription factor (Arabidopsis thaliana) & $1 e-37$ \\
\hline LE30P05 & SGN-U568620 & -2.009 & No hits & No hits & \\
\hline LE20N23 & SGN-U568929 & -2.058 & CAA43171 & TDR6 (Solanum lycopersicum) & $4 e-123$ \\
\hline LE22O19 & SGN-U580463 & -2.148 & P05118 & Wound-induced proteinase inhibitor 1 & $1 e-56$ \\
\hline LE32K23 & SGN-U568929 & -2.269 & CAA43171 & TDR6 (Solanum lycopersicum) & $4 e-123$ \\
\hline LE5A07 & SGN-U579381 & -2.643 & BAA94287 & MADS-box protein pMADS4 (Petunia x hybrida) & $7 e-108$ \\
\hline LE27C20 & SGN-U591985 & -2.742 & O22456 & Developmental protein SEPALLATA 3 & $4 e-28$ \\
\hline LE7J23 & SGN-U577905 & -2.835 & AF448521 & Lycopersicon esculentum MADS-box transcription factor MADS-MC (MADS-MC) & $1 e-124$ \\
\hline LE32I09 & SGN-U577258 & -2.966 & AAA80496 & Flower-specific gamma-thionin-like protein (Solanum lycopersicum) & $3 e-37$ \\
\hline LE8D20 & SGN-U581481 & -5.326 & CAC83066 & MADS-box protein TM29 (Solanum lycopersicum) & $6 e-123$ \\
\hline LE31P14 & SGN-U577283 & -5.681 & NP_200507 & Jasmonic-induced protein 21 (JIP21) (Solanum lycopersicum) & $2 e-118$ \\
\hline LE15K15 & SGN-U585985 & -5.742 & Q9LTS3 & Cytokinin dehydrogenase 3 (CKX3) (Arabidopsis thaliana) & $5 e-48$ \\
\hline LE26B03 & SGN-U572646 & -9.556 & AAW83046 & CRABS CLAW (Nicotiana tabacum) & $1 e-65$ \\
\hline LE1F12 & SGN-U569398 & -13.416 & P48007 & Floral homeotic protein PISTILLATA (GLO1) & $4 e-52$ \\
\hline
\end{tabular}

stages (Figure 4). At early stages of flower development $M C$ transcript abundance was slightly lower in the ufd mutant compared to WT (Figure 4A). Later, expression of $M C$ increased significantly from flowering shoot apex stage in both WT and $u f d$, although it was down-regulated in late wild type inflorescences
(IF2 stage) but continued to increase in $u f d$ inflorescences (Figure 4A). In wild type plants, expression of the floral identity genes SL, TAG1, and TAGL1 increased as the inflorescence developed, while in the ufd plants, these genes were strongly repressed independently of the inflorescence developmental stage 

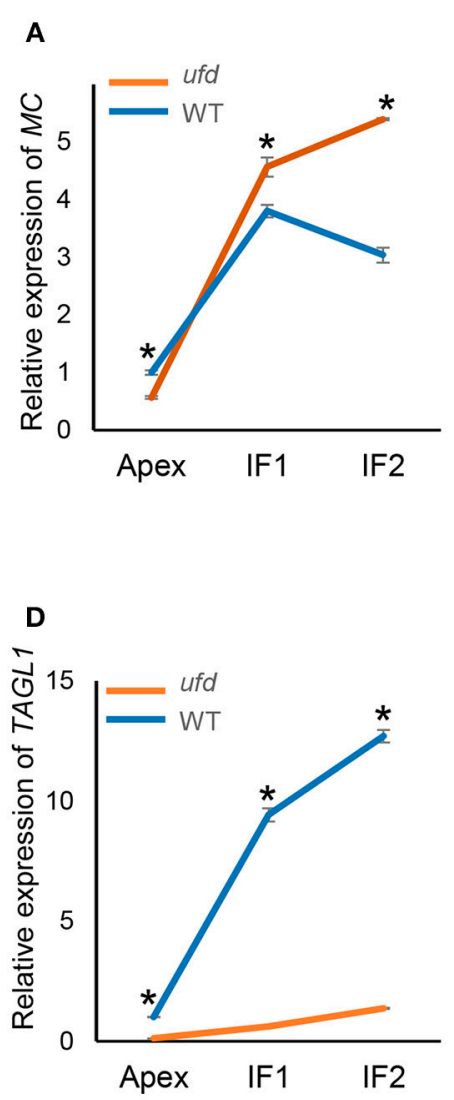

B

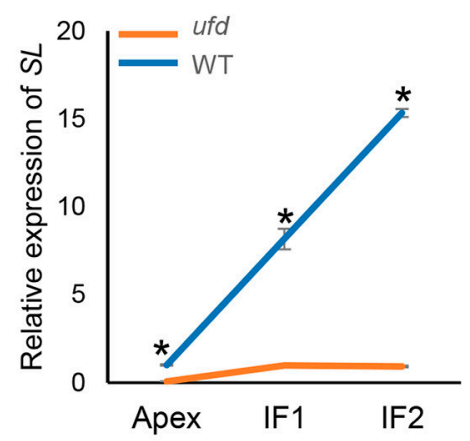

E

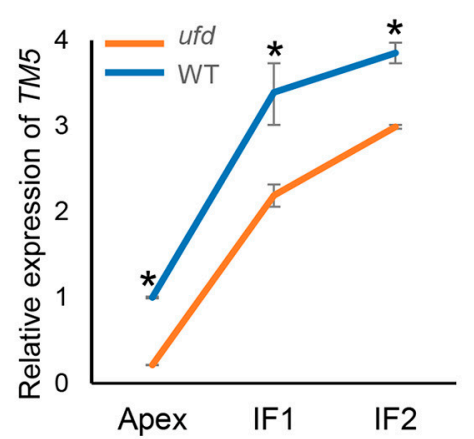

C

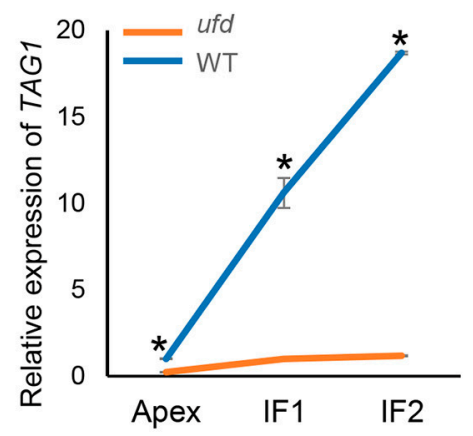

$\mathbf{F}$

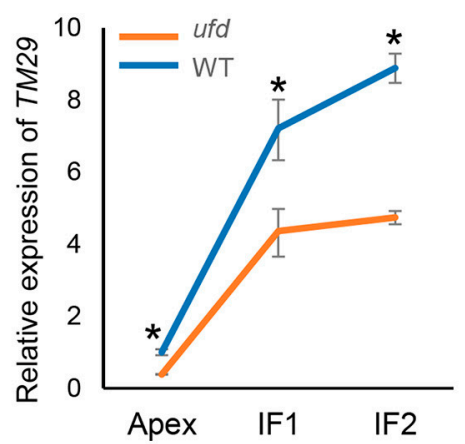

FIGURE 4 | Quantitative real-time PCR determination of expression of (A) MACROCALYX (MC), (B) STAMENLESS (SL), (C) TOMATO AGAMOUS 1 (TAG1), (D) TOMATO AGAMOUS-LIKE 1 (TAGL1), (E) TOMATO MADS BOX 5 (TM5), and (F) TOMATO MADS BOX 29 (TM29) in the wild type (WT) and the unfinished flower development (ufd) mutant flowering inflorescences along their development. Inflorescence developmental stages (i.e., Apex, IF1, and IF2) are described in Material and Methods Section. Error bars show the standard deviation of three independent biological replicates; ${ }^{\star}$ significant differences at $P<0.05$. Panel (A) is from Poyatos-Pertíñez et al. (2016).

(Figures 4B-D). Thus, microarray observations were confirmed since a reduced expression of the B- and C-class genes was found in the ufd mutant compared to the WT. Similarly, TM5 and TM29, whose transcript levels increased during the floral development, were significantly down-regulated in $u f d$ inflorescences (Figures 4E,F).

Likewise, regulatory genes FW2.2, OVATE, and SUN, all of them involved in floral organ growth, were analyzed by qRTPCR (Figure 5). Non-significance differences between WT and ufd were found for these three genes at the apex and young inflorescence (IF1) stages; however, they were differentially expressed at IF2 stage $(P<0.05)$. Thereby, the negative regulators of cell division FW2.2 and OVATE were both overexpressed in ufd compared with wild type plants (Figures $\mathbf{5 A}, \mathbf{B}$ ), whereas the expression of SUN, a positive regulator of organ growth, was repressed in the $u f d$ mutant plants (Figure 5C).

\section{Endogenous Hormone Concentration in the ufd Mutant}

Microarray results indicated that $u f d$ mutation affected transcriptional activity of genes involved in hormone signaling. In order to check if hormone content was also altered in ufd mutant plants, we investigated the hormonal profile in ufd flowering shoot apices. The ufd mutation did not affect the abscisic acid (Figure 6A), salicylic acid (Figure 6C), the jasmonate (Figure 6D) and cytokinins (Figure 6H) concentrations of tomato apices. The strigol (Figure 6F) and ACC (Figure 6G) concentrations were higher in the apices of the $u f d$ mutant compared to the wild type. Differences between genotypes also depended on the hormone forms. Regarding the auxins, no significant differences between genotypes were observed for the total auxin concentration (Figure 6B) but free indole-3-acetic acid (IAA, $128 \pm 14$ vs. $72 \pm 9 \mathrm{pmol} / \mathrm{g}$ ) and conjugated IAA ( $15 \pm 2$ vs. $8 \pm 0.4 \mathrm{pmol} / \mathrm{g}$ and $2.8 \pm 0.2$ vs. 1.1 $\pm 0.1 \mathrm{pmol} / \mathrm{g}$ for IAA-aspartate and IAA-glutamate, respectively) were detected at higher concentration in $u f d$ than wild type flowering apices $(P<0.05$; Supplementary Table 6$)$. On the same way, total gibberellin content did not differ between genotypes (Figure 6E) but the concentration of GA19 was higher in the $u f d$ mutant $(7.4 \pm 0.4$ vs. $5.7 \pm 0.4 \mathrm{pmol} / \mathrm{g}, P<0.05$; Supplementary Table 6). At the cytokinins level, the main significant differences between genotypes were observed for the phosphate forms of trans-zeatin (trans-zeatin riboside monophosphate, $P<0.0001$ ) and cis-zeatin (cis-zeatin riboside monophosphate, $P<0.001$ ), 
A

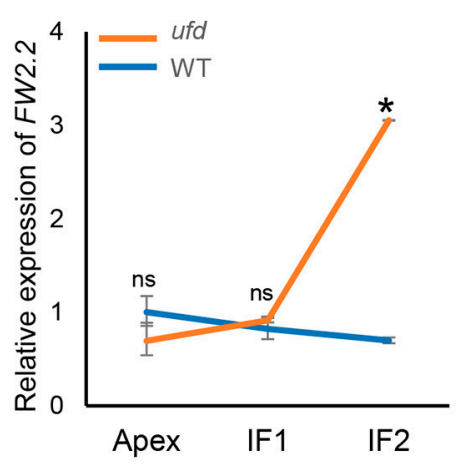

B

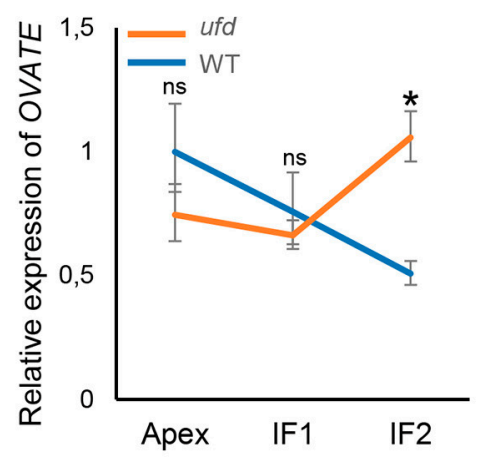

C

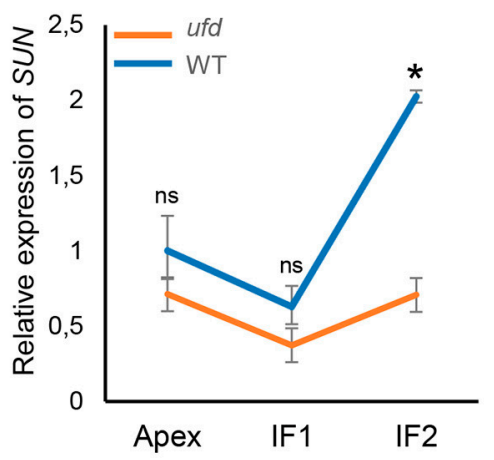

FIGURE 5 | Quantitative real-time PCR determination of expression of (A) FRUIT WEIGHT 2.2 (FW2.2), (B) OVATE (C), and SUN in the wild type (WT) and the unfinished flower development (ufd) mutant flowering inflorescences along their development. Inflorescence developmental stages (i.e., Apex, IF1, and IF2) are described in Material and Methods Section. Error bars show the standard deviation of three independent biological replicates; ns, no statistically significant differences, * significant differences at $P<0.05$.

which were respectively more than three $(11.0 \pm 1.2$ vs. $3.4 \pm$ $0.6 \mathrm{pmol} / \mathrm{g})$ and two times $(2.7 \pm 0.2 \mathrm{vs.} 1.2 \pm 0.3 \mathrm{pmol} / \mathrm{g})$ higher in the $u f d$ mutant compared to WT (Supplementary Table 6).

\section{DISCUSSION}

\section{The ufd Mutation Affects Floral Organ Growth}

The tomato $u f d$ mutant has been identified by screening an EMS-mutagenized M2 population of tomato (cv. Moneymaker), and further characterized in this study. This mutant shows a singular phenotype that affects the growth of floral organs, in such way that they are unable to complete their development. Consequently, flower development is blocked at early stage (stage 5-6 according to Brukhin et al., 2003), when all floral organ primordia have been initiated in their appropriate whorls. The screening of a large segregating population proved that the ufd mutation is inherited as recessive and it does not affect other vegetative or reproductive traits. The UFD function is thus restricted to floral organogenesis, a developmental process where UFD is crucial for making fully developed floral organs, although it is not required for the proper initiation of floral organ primordia.

Mutations in genes affecting floral organ identity and growth usually resulted in homeotic conversion of floral whorls or in abnormal development of the floral organs but did not stop their development at primordia stage (for a review, see Lozano et al., 2009; Causier et al., 2010; Mizzotti et al., 2014; Lombardo and Yoshida, 2015). Even a total loss of floral organ identity in Arabidopsis flowers resulted in the conversion of sepals, petals, stamens, and carpels in leaf-like organs as observed in the sep14 quadruple mutant or in mutants combining mutations in all floral homeotic genes (Bowman et al., 1991; Pelaz et al., 2000; Ditta et al., 2004). In tomato, floral organ identity was partially or completely lost when representative genes of MADS-box classes A $(M C), \mathrm{B}(S L, T M 6, T P I$ and TPIB), C (TAG1), and E (TM5 and TM29) were mutated or down-regulated (Lozano et al., 2009;
Geuten and Irish, 2010; Quinet et al., 2014; Yuste-Lisbona et al., 2016), promoting homeotic changes in the floral organs where these gene functions are required. Additional floral whorls or floral organs were also observed in antisense TM5 plants and ectopic shoots with partially developed leaves and secondary flowers emerged from the fruit in antisense TM29 plants (Pnueli et al., 1994b; Ampomah-Dwamena et al., 2002). Furthermore, tomato mutations that altered regulatory genes involved in the activity and size of the FM as INFLORESCENCE MERISTEM ACTIVITY and the tomato homologous to CLAVATA genes have recently been isolated and further characterized (Sicard et al., 2008; Xu et al., 2015). Once again, the corresponding mutations affected inflorescence architecture and the number of floral organs, although alterations in floral organ identity and growth were not reported. To our knowledge, no other single mutants similar to ufd have been previously reported in tomato or in other model plant species such as Arabidopsis, Antirrhinum, and rice. Only the flowers of the Arabidopsis arf6 arf8 double mutant showed a similar phenotype to the ufd mutant. Thus, arf6 and arf8 single mutants displayed a delay in both stamen filament elongation and anther dehiscence, which reduced selfpollination, whereas floral organ growth was arrested in arf6 arf8 double mutant resulting in the formation of infertile closed buds (Nagpal et al., 2005). However, arf6 arf8 floral buds were blocked after carpel primordia were initiated, at a later developmental stage compared to $u f d$ floral buds (Figure $2 \mathbf{H}$ ). Therefore, it suggests that characterization of $u f d$ mutation could reveal a regulatory gene specifically required for the normal growth of floral organs in tomato. Hopefully, the cloning of UFD gene, which is currently underway, will shed light on its function during flower development.

\section{UFD Affects the Expression of Floral Organ Identity and Growth Genes during Tomato Flower Development}

It is generally accepted that once the FM has been specified, FM identity genes trigger floral organ identity genes, which 


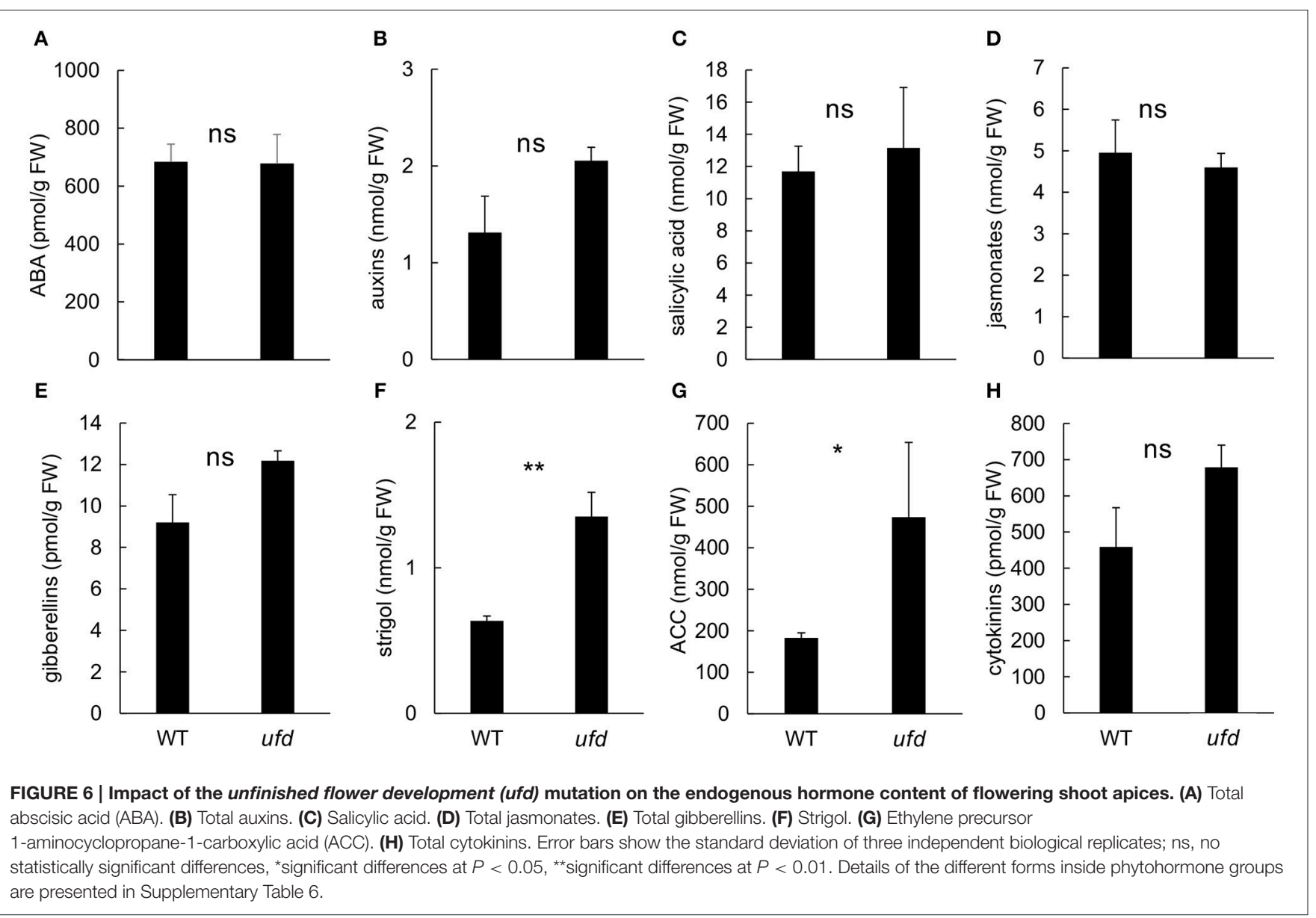

in turn determine floral organ patterning according to the ABC(DE) model (reviewed in Ó'Maoiléidigh et al., 2014; Wellmer et al., 2014). However, after floral organ primordia are initiated, identity, and growth of differentiated cells must be specifically coordinated to promote a full development of floral organs. Expression levels of ABC genes are slightly lower in $u f d$ mutant plants at the onset of flower development, but later during flower development B- and C-class genes were significantly repressed. Previous reports have proved that the $\mathrm{ABC}$ genes play pivotal roles not only determining floral organ identity at early stages of flower development but they are expressed during late stages of floral organogenesis indicating that their functions are needed throughout floral development. Moreover, several pieces of evidence have highlighted the close link between the identity and the final size of floral organs at the end stages of flower development (reviewed in Dornelas et al., 2011; Wellmer et al., 2014). However, genetic and molecular mechanisms coordinating differentiation and growth of specific cells that constitute floral organs still remain unknown (Dornelas et al., 2011). Results reported in the present study suggest that UFD gene may be involved in the genetic network linking both developmental processes in tomato. Transcriptomic and expression analyses performed in $u f d$ mutant showed that the expression levels of $\mathrm{ABC}$ identity genes were smaller than those observed in WT plants during late stages of flower development, suggesting that floral organ primordia, which are unable to grow and complete their development, do not maintain their identity. Furthermore, the failure to grow floral organ primordia in $u f d$ plants is supported by the up-regulation of genes involved in the repression of cell division, as FW2.2 and OVATE. Accordingly, SUN is downregulated in $u f d$ inflorescences as expected from a positive regulator of floral organ growth. Overall, the results obtained suggested that identity and growth are parts of the same developmental program leading to floral organ formation, and that UFD may regulate both $\mathrm{ABC}$ and cell division genes. It is also reasonable to think that organ cells have lost their identity due to their incapacity to proliferate and grow, and therefore, UFD could be a target of ABC identity genes. Under this genetic scenario, UFD would participate in a positive feed-back regulatory pathway enabling the maintenance of the identity of a given floral organ and the differentiation and growth of specific cells composing this organ. Such a hypothesis would be in accordance with the autoregulatory feedback mechanism, which involves MADS-box and other interacting transcription factors (Dornelas et al., 2011). However, other hierarchical relationships between UFD and organ identity genes cannot be discarded until UFD is cloned and its 
functional role in establishing floral organ identity is further determined.

Expression results proved that UFD gene is required for the transcriptional activity of the B-, C-, and E-class genes leading to further development of the floral organ primordia mainly in the three inner whorls. Interestingly, expression of $M C$ in ufd floral buds did not differ significantly from that of WT up until the late stages of inflorescence development, when $M C$ was up-regulated. In addition, sepals of ufd floral buds displayed a normal development and appeared to be more elongated than the remaining floral organs when they were initiated, however their growth was also arrested at the beginning of floral organogenesis. Sepals were suggested as the default state of floral organs and it was proposed that the activities of the FM identity genes during the specification of floral meristems could be involved in specifying sepal identity (Causier et al., 2010; Wellmer et al., 2014). It is true that the first whorl of tomato flowers seems to have a special status since mutations in $M C$ only change sepal identity (Vrebalov et al., 2002; Yuste-Lisbona et al., 2016) while mutations of B- and C-class genes affect floral organs of two consecutive floral whorls and loss-of-function of E-class genes alter the three inner whorls (Pnueli et al., 1994b; Ampomah-Dwamena et al., 2002). In this context, UFD expression should be differently regulated in sepals and in the three innermost floral whorls. Our qRT-PCR results confirmed that $M C$ expression was observed in ufd mutant while B-, C-, and E-class genes remained down-regulated during inflorescence and flower development. Moreover, $M C$ expression continued to increase during inflorescence development in the ufd mutant while it decreased in the WT. That UFD represses the MC expression during floral organ development and activates the B- and C-class genes for proper development of petals, stamens, and carpels should not be excluded.

\section{UFD Participates in the Hormone Signaling Pathways Involved in Floral Development}

The interaction among different phytohormones is thought to mediate the formation of organ primordia and the differentiation of floral organs (Chandler, 2011; Wellmer et al., 2014). In accordance, transcriptomic analyses in Arabidopsis revealed that genes involved in FM and floral organ identity as well as in cell division regulate the expression of many genes involved in hormone responses and metabolism (Kaufmann et al., 2009, 2010; Yant et al., 2010; Wuest et al., 2012; Ó'Maoiléidigh et al., 2013; Winter et al., 2015). In the same way, our results on transcriptome profile showed that genes related to jasmonic acid, cytokinin, and auxin signaling were down-regulated in $u f d$ mutant inflorescences while genes involved in ethylene response were up-regulated suggesting that hormone response could be mediated by UFD transcriptional activity. In tomato plants, insensitivity to hormones often resulted in floral organ defects. Jasmonic acid insensitivity leads to a female sterile phenotype and defects in stamen development in the jai1-1 mutant (Li et al., 2004). This phenotype is partly due to a premature expression of ethylene signaling regulators showing that jasmonic acid and ethylene could have an antagonist effect during flower development (Dobritzsch et al., 2015). Down-regulation of ARF6 and ARF8 resulted in shortened petals, stamens and styles and female sterility (Liu et al., 2014), some of these phenotypes being also attributed to reduced jasmonate production or signaling (Nagpal et al., 2005). Nevertheless, the way in which floral transcription factors and hormones interact to control floral organ growth remained largely unknown. Recently, it has been suggested that SEPALLATA 3 (SEP3) could regulate floral organ development by modulating auxin response in Arabidopsis (Kaufmann et al., 2009). SEP3 belongs to SEP subfamily of MADS-box genes needed to specify the identity of FM and floral organs through the formation of multimeric complexes with ABC proteins (Immink et al., 2009). Therefore, SEP3 could be a linking factor between identity and hormonal signals required for floral organ growth. The fact that expression of TM29, a tomato member of SEP family, is severely repressed in UFD would support this hypothesis.

Moreover, our results showed that the ufd mutation not only affects the expression of genes involved in hormone response but also affects the inflorescence hormonal profile. The production of ACC, strigol, and some forms of auxins, gibberellins, and cytokinins were increased in ufd compared to WT. Modification of the hormonal profile was previously reported in B-class tomato mutants $s l$ and $s l-2$ (Sawhney, 1974; Singh et al., 1992; Singh and Sawhney, 1998, Quinet et al., 2014). These observations might indicate that hormones play a role in proper development of floral organs. In Arabidopsis, the initiation and outgrowth of floral primordia and floral organ initiation depend on the activity of several phytohormones, mainly auxins (Chandler, 2011; Wellmer et al., 2014). Cytokinins and gibberellins also interact with auxins at this level, e.g., in the determination of boundaries between floral organ primordia (Ding et al., 2015). Involvement of these hormones at the early stage of flower development could explain the higher concentration of IAA and some cytokinin forms in the ufd mutant flowering apices. Strigolactones were recently shown to play a hormonal role in plant development (de Saint Germain et al., 2013) and the high strigol concentration in the ufd mutant flowering shoot apex suggests a potential role in flower development in tomato. Further investigations will be required to unravel the hormonal crosstalk underlying floral organ growth in tomato and how it interacts with UFD.

Summarizing, UFD is a regulator of the tomato floral organogenesis that ensures the proper development of floral organ primordia by affecting the expression of $\mathrm{ABC}(\mathrm{DE})$ transcription factors and genes involved in cell division and hormone pathways.

\section{AUTHOR CONTRIBUTIONS}

SP and RL were involved in experimental design and interpretation of data. SP, MQ, AO, and EG. performed experiments and analyzed data; SP, MQ, FY, and RL wrote, reviewed and edited the manuscript; $\mathrm{CP}$ and AG supported microarray analysis and data handling; JC and TA supervised 
experiments and provided critical review and editing of the manuscript. All authors contributed to editing and approving the final version of the manuscript.

\section{FUNDING}

This research was supported by the Spanish Ministry of Economy and Competitiveness and the EU European Regional Development Fund (Grants BIO2009-11484 and AGL2015-64991-C3-R-1). We also thank Campus de Excelencia Internacional Agroalimentario (CeiA3) for providing financial support. $\mathrm{AO}$ is a recipient of a $\mathrm{PhD}$ fellowship from the Ministerio de Ciencia e Innovación of Spain (BIO2009-11484).

\section{REFERENCES}

Ampomah-Dwamena, C., Morris, B. A., Sutherland, P., Veit, B., and Yao, J. L. (2002). Down-regulation of TM29, a tomato SEPALLATA homolog, causes parthenocarpic fruit development and floral reversion. Plant Physiol. 130, 605-617. doi: 10.1104/pp.005223

Anastasiou, E., and Lenhard, M. (2007). Growing up to one's standard. Curr. Opin. Plant Biol. 10, 63-69. doi: 10.1016/j.pbi.2006.11.002

Azzi, L., Deluche, C., Gévaudant, F., Frangne, N., Delmas, F., Hernould, M., et al. (2015). Fruit growth-related genes in tomato. J. Exp. Bot. 66, 1075-1086. doi: 10.1093/jxb/eru527

Bögre, L., Magyar, Z., and López-Juez, E. (2008). New clues to organ size control in plants. Genome Biol. 9:226. doi: 10.1186/gb-2008-9-7-226

Bowman, J. L., Drews, G. N., and Meyerowitz, E. M. (1991). Expression of the Arabidopsis floral homeotic gene AGAMOUS is restricted to specific cell types late in flower development. Plant Cell 3, 749-758. doi: 10.1105/tpc.3.8.749

Brukhin, V., Hernould, M., Gonzalez, N., Chevalier, C., and Mouras, A. (2003). Flower development schedule in tomato Lycopersicon esculentum cv. sweet cherry. Sex. Plant Reprod. 15, 311-320. doi: 10.1007/s00497-003-0167-7

Busi, M. V., Bustamante, C., D’Angelo, C., Hidalgo-Cuevas, M., Boggio, S. B., Valle, E. M., et al. (2003). MADS-box genes expressed during tomato seed and fruit development. Plant Mol. Biol. 52, 801-815. doi: 10.1023/A:1025001402838

Causier, B., Schwarz-Sommer, Z., and Davies, B. (2010). Floral organ identity: 20 years of ABCs. Semin. Cell Dev. Biol. 21, 73-79. doi: 10.1016/j.semcdb.2009.10.005

Chandler, J. W. (2011). The hormonal regulation of flower development. J. Plant Growth Regul. 30, 242-254. doi: 10.1007/s00344-010-9180-x

Coen, E. S., and Meyerowitz, E. M. (1991). The war of the whorls: genetic interactions controlling flower development. Nature 353, 31-37. doi: 10.1038/353031a0

de Martino, G., Pan, I., Emmanuel, E., Levy, A., and Irish, V. F. (2006). Functional analyses of two tomato APETALA3 genes demonstrate diversification in their roles in regulating floral development. Plant Cell 18, 1833-1845. doi: 10.1105/tpc.106.042978

de Saint Germain, A., Bonhomme, S., Boyer, F. D., and Rameau, C. (2013). Novel insights into strigolactone distribution and signalling. Curr. Opin. Plant Biol. 16, 583-589. doi: 10.1016/j.pbi.2013.06.007

Ding, L., Yan, S., Jiang, L., Zhao, W., Ning, K., Zhao, J., et al. (2015). HANABA TARANU (HAN) bridges meristem and organ primordia boundaries through PINHEAD, JAGGED, BLADE-ON-PETIOLE2 and CYTOKININ OXIDASE 3 during flower development in Arabidopsis. PLoS Genet. 11:e1005479. doi: 10.1371/journal.pgen.1005479

Ditta, G., Pinyopich, A., Robles, P., Pelaz, S., and Yanofsky, M. F. (2004). The SEP4 gene of Arabidopsis thaliana functions in floral organ and meristem identity. Curr. Biol. 14, 1935-1940. doi: 10.1016/j.cub.2004.10.028

Dobrev, P. I., and Kamínek, M. (2002). Fast and efficient separation of cytokinins from auxin and abscisic acid and their purification using mixed-mode solid phase extraction. J. Chromatogr. A 950, 21-29. doi: 10.1016/S00219673(02)00024-9

\section{ACKNOWLEDGMENTS}

Seeds of accessions LA2706 (S. lycopersicum L., cultivar Moneymaker) and LA1589 (S. pimpinellifolium) were provided by the Tomato Genetic Resource Center (TGRC, University of California, Davis). The authors thank Vaclav Motyka and Peter Dobrev for phytohormonal analysis.

\section{SUPPLEMENTARY MATERIAL}

The Supplementary Material for this article can be found online at: http://journal.frontiersin.org/article/10.3389/fpls.2016. 01648/full\#supplementary-material

Dobritzsch, S., Weyhe, M., Schubert, R., Dindas, J., Hause, G., Kopka, J., et al. (2015). Dissection of jasmonate functions in tomato stamen development by transcriptome and metabolome analyses. BMC Biol. 13:28. doi: 10.1186/s12915015-0135-3

Dornelas, M. C., Patreze, C. M., Angenent, G. C., and Immink, R. G. M. (2011). MADS: the missing link between identity and growth? Trends Plant Sci. 16, 89-97. doi: 10.1016/j.tplants.2010.11.003

Ó'Maoiléidigh, D. S., Graciet, E., and Wellmer, F. (2014). Gene networks controlling Arabidopsis thaliana flower development. New Phytol. 201, 16-30. doi: $10.1111 /$ nph. 12444

Ó’Maoiléidigh, D. S., Wuest, S. E., Rae, L., Raganelli, A., Ryan, P. T., Kwasniewska, K., et al. (2013). Control of reproductive floral organ identity specification in Arabidopsis by the C function regulator AGAMOUS. Plant Cell 25, 2482-2503. doi: 10.1105/tpc.113.113209

Frary, A., Nesbitt, T. C., Frary, A., Grandillo, S., van der Knaap, E., Cong, B., et al. (2000). fw2.2: a quantitative trait locus key to the evolution of tomato fruit size. Science 289, 85-88. doi: 10.1126/science.289.5476.85

Gallego-Giraldo, C., Hu, J., Urbez, C., Gomez, M. D., Sun, T. P., and PerezAmador, M. A. (2014). Role of the gibberellin receptors GID1 during fruit-set in Arabidopsis. Plant J. 79, 1020-1032. doi: 10.1111/tpj.12603

Geuten, K., and Irish, V. (2010). Hidden variability of floral homeotic B genes in Solanaceae provides a molecular basis for the evolution of novel functions. Plant Cell 22, 2562-2578. doi: 10.1105/tpc.110.076026

Giménez, E., Pineda, B., Capel, J., Antón, M. T., Atarés, A., Pérez-Martín, F., et al. (2010). Functional analysis of the Arlequin mutant corroborates the essential role of the ARLEQUIN/TAGL1 gene during reproductive development of tomato. PLoS ONE 5:e14427. doi: 10.1371/journal.pone.0014427

Hackbusch, J., Richter, K., Müller, J., Salamini, F., and Uhrig, J. F. (2005). A central role of Arabidopsis thaliana ovate family proteins in networking and subcellular localization of 3-aa loop extension homeodomain proteins. Proc. Natl. Acad. Sci. U.S.A. 102, 4908-4912. doi: 10.1073/pnas.0501181102

Honma, T., and Goto, K. (2001). Complexes of MADS-box proteins are sufficient to convert leaves into floral organs. Nature 409, 525-529. doi: 10.1038/35054083

Immink, R. G., Tonaco, I. A., de Folter, S., Shchennikova, A., van Dijk, A. D., Busscher-Lange, J., et al. (2009). SEPALLATA3: the 'glue' for MADS box transcription factor complex formation. Genome Biol. 10:R24. doi: 10.1186/gb2009-10-2-r24

Jaeger, K. E., Pullen, N., Lamzin, S., Morris, R. J., and Wigge, P. A. (2013). Interlocking feedback loops govern the dynamic behavior of the floral transition in Arabidopsis. Plant Cell 25, 820-833. doi: 10.1105/tpc.113.109355

Kaufmann, K., Muiño, J. M., Jauregui, R., Airoldi, C. A., Smaczniak, C., Krajewski, P., et al. (2009). Target genes of the MADS transcription factor SEPALLATA3: integration of developmental and hormonal pathways in the Arabidopsis flower. PLoS Biol. 7:e1000090. doi: 10.1371/journal.pbio.1000090

Kaufmann, K., Wellmer, F., Muiño, J. M., Ferrier, T., Wuest, S. E., Kumar, V., et al. (2010). Orchestration of floral initiation by APETALA1. Science 328, 85-89. doi: $10.1126 /$ science. 1185244

Kramer, E. M., Dorit, R. L., and Irish, V. F. (1998). Molecular evolution of genes controlling petal and stamen development: duplication and divergence 
within the APETALA3 and PISTILLATA MADS-box gene lineages. Genetics 149, 765-783.

Leseberg, C. H., Eissler, C. L., Wang, X., Johns, M. A., Duvall, M. R., and Mao, L. (2008). Interaction study of MADS-domain proteins in tomato. J. Exp. Bot. 59, 2253-2265. doi: 10.1093/jxb/ern094

Li, L., McCaig, B. C., Wingerd, B. A., Wang, J., Whaton, M., Pichersky, E., et al. (2004). The tomato homolog of CORONATINE-INSENSITIVE1 is required for the maternal control of seed maturation, jasmonate-signaled defense responses, and glandular trichome development. Plant Cell 16, 126-143. doi: 10.1105/tpc.017954

Liu, J., Van Eck, J., Cong, B., and Tanksley, S. D. (2002). A new class of regulatory genes underlying the cause of pear-shaped tomato fruit. Proc. Natl. Acad. Sci. U.S.A. 99, 13302-13306. doi: 10.1073/pnas.162485999

Liu, N., Wu, S., Van Houten, J., Wang, Y., Ding, B., Fei, Z., et al. (2014). Downregulation of AUXIN RESPONSE FACTORS 6 and 8 by microRNA 167 leads to floral development defects and female sterility in tomato. J. Exp. Bot. 65, 2507-2520. doi: 10.1093/jxb/eru141

Lombardo, F., and Yoshida, H. (2015). Interpreting lemma and palea homologies: a point of view from rice floral mutants. Front. Plant Sci. 6:61. doi: $10.3389 /$ fpls.2015.00061

Lozano, R., Giménez, E., Cara, B., Capel, J., and Angosto, T. (2009). Genetic analysis of reproductive development in tomato. Int. J. Dev. Biol. 53, 1635-1648. doi: $10.1387 / \mathrm{ijdb} .072440 \mathrm{rl}$

Lytovchenko, A., Eickmeier, I., Pons, C., Osorio, S., Szecowka, M., Lehmberg, K., et al. (2011). Tomato fruit photosynthesis is seemingly unimportant in primary metabolism and ripening but plays a considerable role in seed development. Plant Physiol. 157, 1650-1663. doi: 10.1104/pp.111.186874

Marsch-Martínez, N., and de Folter, S. (2016). Hormonal control of the development of the gynoecium. Curr. Opin. Plant Biol. 29, 104-114. doi: 10.1016/j.pbi.2015.12.006

Mizzotti, C., Galliania, B. M., and Masieroa, S. (2014). The backstage of the ABC model: the Antirrhinum majus contribution. Plant Biosyst. 148, 176-186. doi: 10.1080/11263504.2013.877531

Molinero-Rosales, N., Jamilena, M., Zurita, S., Gómez, P., Capel, J., and Lozano, R. (1999). FALSIFLORA, the tomato orthologue of FLORICAULA and LEAFY, controls flowering time and floral meristem identity. Plant J. 20, 685-693. doi: 10.1046/j.1365-313X.1999.00641.x

Nagpal, P., Ellis, C. M., Weber, H., Ploense, S. E., Barkawi, L. S., Guilfoyle, T. J., et al. (2005). Auxin response factors ARF6 and ARF8 promote jasmonic acid production and flower maturation. Development 132, 4107-4118. doi: $10.1242 / \operatorname{dev} .01955$

Pajoro, A., Madrigal, P., Muiño, J. M., Matus, J. T., Jin, J., Mecchia, M. A., et al. (2014). Dynamics of chromatin accessibility and gene regulation by MADSdomain transcription factors in flower development. Genome Biol. 15:R41. doi: 10.1186/gb-2014-15-3-r41

Pelaz, S., Ditta, G. S., Baumann, E., Wisman, E., and Yanofsky, M. F. (2000). B and C floral organ identity functions require SEPALLATA MADS-box genes. Nature 405, 200-203. doi: 10.1038/35012103

Pesaresi, P., Mizzotti, C., Colombo, M., and Masiero, S. (2014). Genetic regulation and structural changes during tomato fruit development and ripening. Front. Plant Sci. 5:124. doi: 10.3389/fpls.2014.00124

Plackett, A. R., Thomas, S. G., Wilson, Z. A., and Hedden, P. (2011). Gibberellin control of stamen development: a fertile field. Trends Plant Sci. 16, 568-578. doi: 10.1016/j.tplants.2011.06.007

Pnueli, L., Abu-Abeid, M., Zamir, D., Nacken, W., Schwarz-Sommer, Z., and Lifschitz, E. (1991). The MADS box gene family in tomato: temporal expression during floral development, conserved secondary structures and homology with homeotic genes from Antirrhinum and Arabidopsis. Plant J. 1, 255-266. doi: 10.1111/j.1365-313X.1991.00255.x

Pnueli, L., Hareven, D., Broday, L., Hurwitz, C., and Lifschitz, E. (1994b). The TM5 MADS box gene mediates organ differentiation in the three inner whorls of tomato flowers. Plant Cell 6, 175-186. doi: 10.1105/tpc.6.2.175

Pnueli, L., Hareven, D., Rounsley, S., Yanofsky, M., and Lifschitz, E. (1994a). Isolation of the tomato AGAMOUS gene TAG1 and analysis of its homeotic role in transgenic plants. Plant Cell 6, 163-173. doi: 10.1105/tpc.6.2.163

Poyatos-Pertínez, S., Quinet, M., Ortíz-Atienza, A., Bretones, S., Yuste-Lisbona, F. J., and Lozano, R. (2016). Genetic interactions of the unfinished flower development (ufd) mutant support a significant role of the tomato UFD gene in regulating floral organogenesis. Plant Reprod. 29, 227-238. doi: 10.1007/s00497-016-0286-6

Quinet, M., Bataille, G., Dobrev, P. I., Capel, C., Gómez, P., Capel, J., et al. (2014). Transcriptional and hormonal regulation of petal and stamen development by STAMENLESS, the tomato (Solanum lycopersicum L.) orthologue to the B-class APETALA3 gene. J. Exp. Bot. 65, 2243-2256. doi: 10.1093/jxb/eru089

Reinhardt, D. (2005). Regulation of phyllotaxis. Int. J. Dev. Biol. 49, 539-546. doi: $10.1387 / \mathrm{ijdb} .041922 \mathrm{dr}$

Sawhney, V. K. (1974). Morphogenesis of the stamenless-2 mutant in tomato. III. Relative levels of gibberellins in the normal and mutant plants. J. Exp. Bot. 25, 1004-1009. doi: 10.1093/jxb/25.6.1004

Sicard, A., Petit, J., Mouras, A., Chevalier, C., and Hernould, M. (2008). Meristem activity during flower and ovule development is controlled by the mini zinc finger gene INHIBITOR OF MERISTEM ACTIVITY in tomato. Plant J. 55, 415-427. doi: 10.1111/j.1365-313X.2008.03520.x

Singh, S., and Sawhney, V. K. (1998). Abscisic acid in a male sterile tomato mutant and its regulation by low temperature. J. Exp. Bot. 49, 199-203. doi: $10.1093 / \mathrm{jxb} / 49.319 .199$

Singh, S., Sawhney, V. K., and Pearce, D. W. (1992). Temperature effects on endogenous indole-3-acetic acid levels in leaves and stamens of the normal and male sterile 'stamenless-2' mutant of tomato (Lycopersicon esculentum Mill.). Plant Cell Environ. 15, 373-377. doi: 10.1111/j.1365-3040.1992.tb0 0986.x

Siriwardana, N. S., and Lamb, R. S. (2012). The poetry of reproduction: the role of LEAFY in Arabidopsis thaliana flower formation. Int. J. Dev. Biol. 56, 207-221. doi: $10.1387 / \mathrm{ijdb} .113450 \mathrm{~ns}$

Smaczniak, C., Immink, R. G., Muino, J. M., Blanvillain, R., Busscher, M., Busscher-Lange, J., et al. (2012). Characterization of MADS-domain transcription factor complexes in Arabidopsis flower development. Proc. Natl. Acad. Sci. U.S.A. 109, 1560-1565. doi: 10.1073/pnas.1112871109

Thouet, J., Quinet, M., Lutts, S., Kinet, J. M., and Périlleux, C. (2012). Repression of floral meristem fate is crucial in shaping tomato inflorescence. PLOS ONE 7:e31096. doi: 10.1371/journal.pone.0031096

Vrebalov, J., Pan, I. L., Arroyo, A. J. M., McQuinn, R., Chung, M., Poole, M., et al. (2009). Fleshy fruit expansion and ripening are regulated by the tomato SHATTERPROOF gene TAGL1. Plant Cell 21, 3041-3062. doi: 10.1105/tpc.109.066936

Vrebalov, J., Ruezinsky, D., Padmanabhan, V., White, R., Medrano, D., Drake, R., et al. (2002). A MADS-box gene necessary for fruit ripening at the tomato Ripening-Inhibitor (Rin) locus. Science 296, 343-346. doi: $10.1126 /$ science. 1068181

Wang, S., Chang, Y., Guo, J., and Chen, J. G. (2007). Arabidopsis Ovate Family Protein 1 is a transcriptional repressor that suppresses cell elongation. Plant $\mathrm{J}$. 50, 858-872. doi: 10.1111/j.1365-313X.2007.03096.x

Wang, S., Chang, Y., Guo, J., Zeng, Q., Ellis, B. E., and Chen, J. G. (2011). Arabidopsis Ovate family proteins, a novel transcriptional repressor family, control multiple aspects of plant growth and development. PLoS ONE 6:e23896. doi: 10.1371/journal.pone.0023896

Wellmer, F., Alves-Ferreira, M., Dubois, A., Riechmann, J. L., and Meyerowitz, E. M. (2006). Genome-wide analysis of gene expression during early Arabidopsis flower development. PLoS Genet. 2:e117. doi: 10.1371/journal.pgen.0020117

Wellmer, F., Graciet, E., and Riechmann, J. L. (2014). Specification of floral organs in Arabidopsis. J. Exp. Bot. 65, 1-9. doi: 10.1093/jxb/ert385

Wellmer, F., Riechmann, J. L., Alves-Ferreira, M., and Meyerowitz, E. M. (2004). Genome-wide analysis of spatial gene expression in Arabidopsis flowers. Plant Cell 16, 1314-1326. doi: 10.1105/tpc.021741

Winter, C. M., Yamaguchib, N., Wub, M. F., and Wagner, D. (2015). Transcriptional programs regulated by both LEAFY and APETALA1 at the time of flower formation. Physiol. Plant. 155, 55-73. doi: 10.1111/ppl.12357

Wuest, S. E., Ó’Maoiléidigh, D. S., Rae, L., Kwasniewska, K., Raganelli, A., Hanczaryk, K., et al. (2012). Molecular basis for the specification of floral organs by APETALA3 and PISTILLATA. Proc. Natl. Acad. Sci. U.S.A. 109, 13452-13457. doi: 10.1073/pnas.1207075109

Xiao, H., Jiang, N., Schaffner, E. K., Stockinger, E. J., and van der Knaap, E. (2008). A retrotransposon-mediated gene duplication underlies morphological variation of tomato fruit. Science 319, 1527-1530. doi: 10.1126/science.1153040

Xiao, H., Radovich, C., Welty, N., Hsu, J., Li, D., Meulia, T., et al. (2009). Integration of tomato reproductive developmental landmarks and expression 
profiles, and the effect of SUN on fruit shape. BMC Plant Biol. 9:49. doi: 10.1186/1471-2229-9-49

Xu, C., Liberatore, K. L., MacAlister, C. A., Huang, Z., Chu, Y. H., Jiang, K., et al. (2015). A cascade of arabinosyltransferases controls shoot meristem size in tomato. Nat. Genet. 47, 784-792. doi: 10.1038/ ng.3309

Yant, L., Mathieu, J., Dinh, T. T., Ott, F., Lanz, C., Wollmann, H., et al. (2010). Orchestration of the floral transition and floral development in Arabidopsis by the bifunctional transcription factor APETALA2. Plant Cell 22, 2156-2170. doi: 10.1105/tpc.110.075606

Yuan, Z., and Zhang, D. (2015). Roles of jasmonate signalling in plant inflorescence and flower development. Curr. Opin. Plant Biol. 27, 44-51. doi: 10.1016/j.pbi.2015.05.024

Yuste-Lisbona, F. J., Quinet, M., Fernández-Lozano, A., Pineda, B., Moreno, V., Angosto, T., et al. (2016). Characterization of vegetative inflorescence (mc-vin) mutant provides new insight into the role of MACROCALYX in regulating inflorescence development of tomato. Sci. Rep. 6:18796. doi: 10.1038/srep 18796

Conflict of Interest Statement: The authors declare that the research was conducted in the absence of any commercial or financial relationships that could be construed as a potential conflict of interest.

Copyright () 2016 Poyatos-Pertiñez, Quinet, Ortíz-Atienza, Yuste-Lisbona, Pons, Giménez, Angosto, Granell, Capel and Lozano. This is an open-access article distributed under the terms of the Creative Commons Attribution License (CC BY).

The use, distribution or reproduction in other forums is permitted, provided the original author(s) or licensor are credited and that the original publication in this journal is cited, in accordance with accepted academic practice. No use, distribution or reproduction is permitted which does not comply with these terms. 